\title{
Una nuova lettera di Ugo Foscolo
}

Une nouvelle lettre de Ugo Foscolo

A New Letter of Ugo Foscolo

\section{Christian Del Vento}

\section{(2) OpenEdition}

\section{Journals}

\section{Edizione digitale}

URL: http://journals.openedition.org/cei/2367

DOI: $10.4000 /$ cei. 2367

ISSN: 2260-779X

\section{Editore}

UGA Éditions/Université Grenoble Alpes

\section{Edizione cartacea}

Data di pubblicazione: 30 juin 2015

Paginazione: 79-84

ISBN: 978-2-84310-289-9

ISSN: 1770-9571

Notizia bibliografica digitale

Christian Del Vento, «Una nuova lettera di Ugo Foscolo», Cahiers d'études italiennes [Online], 20 | 2015, online dal 01 janvier 2017, consultato il 28 mars 2021. URL: http://journals.openedition.org/cei/2367 DOI: https://doi.org/10.4000/cei.2367 


\title{
UNA NUOVA LETTERA DI UGO FOSCOLO
}

\author{
Christian Del Vento
}

Université Sorbonne Nouvelle - Paris 3

Tra gli autografi della ricchissima collezione di Prosper Tarbé che la figlia legò nel I89I alla Bibliothèque Municipale di Reims ${ }^{\mathrm{I}}$, si conserva (Archives Municipales, Coll. Tarbé, XXIII 4I) anche una lettera inedita di Ugo Foscolo all'abate di Breme, di cui fino ad ora era nota solo la risposta dello scrittore milanese, custodita alla Biblioteca Nazionale Centrale di Firenze (Foscolo, VII R 2) e pubblicata da Plinio Carli nel quinto volume dell'Epistolario foscoliano ${ }^{2}$.

La riproduciamo di seguito:

Monsr. Mr. l'Abbé de Breme.

Le rimando il libro della Filosofessa: non le rincresca di far consegnare al mio ajutante di campo, vulgo servidore, il volume dell'edizione dell'Haine, dov'io possa leggere il $3 .^{20}$ delle Georgiche; lo rimanderò Sabbato mattina, e le chiederò allora il Laocoonte di Lessing; così Ella promoverà i miei studj e con le lodi e co' libri, finchè un giorno esaminando con più quiete le cose ch'io vado scrivendo, Ella mi ajuterà di consigli e di correzioni. - Piacciale di salutare il Conte di Sartirana ${ }^{3}$, e di dare un bacio in mio nome al suo nipotino ${ }^{4}$, affinch'egli non si dimentichi del mio nome. Bene Valeque. -

di casa, Lunedì -

I. Su Louis Hardouin Prosper Tarbé, magistrato, storico e archeologo, e sulla sua ricca collezione si vedano le informazioni che se ne danno nell'introduzione al tomo XXXIX bis del monumentale Catalogue général des manuscrits des bibliothèques publiques de France, Paris, Plon, I909, pp. I-XXII, interamente consacrato a questa collezione.

2. È la lettera ${ }^{\circ}{ }^{\circ} 602$ (Ep. V, pp. 288-289). Plinio Carli datò la risposta dell'abate di Breme all'ottobre I8I4, seguito poi da Piero Camporesi (L. di Breme, Lettere, Torino, Einaudi, 1966, nº II3).

3. Il marchese Ludovico Giuseppe Arborio Gattinara di Sartirana di Breme, padre dell'abate Di Breme, che fu ministro degli interni del Regno d'Italia.

4. Ferdinando di Sartirana. 
L’inglese Craken:` è egli tornato da' laghi?

Suo devotis. ${ }^{\circ}$ ed am. ${ }^{\circ}$

Ugo Foscolo.

Si tratta di una missiva breve ma importante, perché consente di precisare alcune delle letture che lo scrittore fece durante la redazione degli scritti teorici che avrebbero dovuto accompagnare la pubblicazione delle Grazie.

Innanzitutto, il Laocoonte di Lessing, come felicemente congetturato dal Carli e dal Camporesi ${ }^{6}$. La lettera consente ora di confermare ciò che negli ultimi vent'anni alcuni eminenti studiosi delle Grazie hanno ipotizzato ${ }^{7}$ e che, in questo stesso volume, Elena Parrini Cantini dimostra in maniera indiscutibile, almeno per la "forma ultima» del carme, la Dissertation on an Ancient Hymn to the Graces del I822: che nel rifiuto foscoliano del secolare precetto dell'ut pictura poësis, abbia avuto un ruolo decisivo la lettura del trattato di Lessing ${ }^{8}$.

In secondo luogo, Foscolo parla di una «Filosofessa». L'epiteto sarcastico fa riferimento a Madame de Staël: dietro il rinvio alla protagonista del romanzo dell'abate Chiari, una donna italiana ma di origini francesi, che scrive le sue memorie filosofeggiando, si legge in filigrana la figura della musa di Coppet. Più problematica è, invece, l'identificazione del volume. Potrebbe trattarsi, infatti, sia del romanzo Corinne ou l'Italie, che del trattato De l'Allemagne. Gli argomenti a favore dell'una o dell'altra identificazione non mancano. Per quanto concerne De l'Allemagne varrà non solo la prossimità cronologica (il trattato era stato pubblicato nel I8I3, dopo la pesantissima censura cui era stata sottoposta l'edizione del I8IO $^{9}$ ), ma anche l'importanza che il contemporaneo dibattito filosofico ed estetico tedesco vi rivestono, in particolare la figura di Lessing, oggetto di un

5. Si tratta di M. de Crackenthorpe, un nobile inglese che la contessa d'Albany aveva fatto conoscere a Foscolo (Ep. V, n ${ }^{\circ}$ I58I) e questi al di Breme (Ep. V, n ${ }^{\circ}$ i587).

6. A partire dalla risposta del di Breme ("Verrà Lessing ad un minimo suo cenno») il Carli esitava tra il Laocoonte e l'Emilia Gallotti (Ep. V, p. 288n.). Il Camporesi, invece, avanzava l'ipotesi che il volume fosse proprio il Laocoonte.

7. Si vedano gli studi di S. Scatizzi, Il «Laocoonte» di Lessing nella poetica foscoliana: la lettera al Fabre e le "Grazie», «Moderni e Antichi», vol. II-III, 2004-2005, pp. 38I-443; e di R. Cotrone, Ut pictura poësis: arti verbali e arti figurative in Lessing e Foscolo, in G. Baldassarri e S. Tamiozzo (a cura di), Letteratura italiana, letterature europee, Atti del Congresso nazionale dell'ADI (Padova-Venezia, I8-2I settembre 2002), Roma, Bulzoni, 2004, pp. 477-485. La presenza di Lessing dietro la teoria estetica abbozzata da Foscolo nella dedica alla contessa d'Albany era stata già suggerita da Mario Scotti nella sua edizione delle Grazie. Cfr. EN I, p. 1262.

8. A. Bruni, In margine alle "Grazie» inglesi di Foscolo, in Outline Engravings and Descriptions of the Woburn Abbey Marbles (London, Printed by William Nicol Shakespeare Press, Cleveland-How, St. James's, M.DCCC. XXII) / Le Grazie a Woburn Abbey, a cura di A. Bruni, Firenze, Polistampa, 20I2, p. CCXviII.

9. De l'Allemagne, par Mme la baronne de Staël Holstein..., Paris, Nicolle, i81o; Londres, J. Murray, I813, 3 voll. 
celebre confronto con quella di Winckelmann ${ }^{\text {Io }}$. In uno dei molteplici frammenti che costituiscono il dossier genetico delle cosiddette Lettere scritte dall'Inghilterra" ${ }^{\text {II }}$, si legge: "Vedo [...] la Metafisica tedesca rivestita delle gonnelle di Mme de Staël, e predica con l'eloquenza della scuola Ginevrina e con l'entusiasmo degli innamorati per sistema, e corre per l'Europa ed è accolta in ogni parte» ${ }^{12}$; dove, anche se per le Lettere Foscolo si fondò soprattutto su Corinne, si sarebbe tentati di scorgere un riferimento proprio al trattato De l'Allemagne e a una sua possibile lettura da parte dello scrittore.

Qualunque fosse il volume chiesto in prestito a Ludovico di Breme nell'autunno del I8I4, le ragioni dell'interesse manifestato da Foscolo per Madame de Staël all'altezza delle Grazie e della perplessità per chi aveva sposato la causa della filosofia e dell'estetica tedesche si colgono nelle pagine taglienti che le dedica proprio nelle Lettere. Esse si riassumono nell'incapacità della scrittrice a cogliere il segreto di ciò che è grande e bello nella natura e nell'arte, e nella superfetazione sentimentale che questa incomprensione generava. Agli occhi di Foscolo, infatti, Madame de Staël era uno dei più ferventi sostenitori di quella "Metafisica» che aveva «bandita la corte del vecchio Re [Omero] per corteggiare gli spettri del nuovo", "cantando odi italiane in prosa francese e facendo analiticamente all'amore» ${ }^{13}$. Madame de Staël, circuita dalla filosofia idealista, si era illusa di poter «fondare avvenimenti storici sopra la favola», invertendo il rapporto tra finzione e verità e ingannando «sè medesima e gli altri ${ }^{\Gamma 4}$. Si capisce, allora, l'interesse di Foscolo per l'opera della scrittrice proprio mentre si apprestava a scrivere i testi teorici che accompagnavano le Grazie, in cui rivendicava una prospettiva estetica di tipo classicista, la stessa che, anni dopo, ormai in Inghilterra, avrebbe difeso ancora nei Principles of Poetical Criticism as Applicable more Especially to Italian Literature (I824).

Io. La si legge nel capitolo VI del libro primo (De l'Allemagne, Londres, cit., pp. 239-248). Nel terzo libro, consacrato alla filosofia e alla morale tedesche prima e dopo Kant, Madame de Staël torna su Lessing, affiancandolo a Hemsterhuis e a Jacobi.

II. Che si leggono in EN V, pp. 237-454 (pp. LXIV-CXviII per l'introduzione di M. Fubini). Con questo titolo si raccolgono gli esiti di un'opera in forma epistolare alla quale Foscolo si dedicò tra l'estate del I8I7 e i primi mesi del I8I8, ma che restò inedita e incompiuta. Il materiale riguardante le Lettere comprende tutti gli stadi intermedi della composizione, dai primi abbozzi alle belle copie quasi definitive, ma l'ordinamento tanto dei fascicoli quanto dei fogli singoli è del tutto arbitrario e, come ha dimostrato di recente E. Lombardi (Per l'edizione critica delle "Lettere scritte dall'Inghilterra», "Studi di filologia italiana», LIII, 1995, pp. 245-344), neppure Fubini risolse il problema della cronologia interna all'opera.

I2. EN V, p. 375 .

13. Citiamo il testo nell'edizione che ne dà E. Lombardi in U. Foscolo, Opere, ed. diretta da F. Gavazzeni, vol. II, Prose e Saggi, Torino, Einaudi, 1995, pp. 458, 460.

I4. Ibid. 
L'ultimo testo che incontriamo nella lettera è la fondamentale edizione delle Georgiche di Virgilio approntata da Christian Gottlob Heyne, uno studioso che ebbe grande importanza nella formazione del pensiero di Foscolo fin dalla Chioma di Berenice (dove l'edizione è largamente citata) ${ }^{\text {I5 }}$. Anche l'edizione di Heyne servì a Foscolo per redigere l'apparato di testi teorici che accompagna il carme, in particolare l'abbozzo d'introduzione teorica che, probabilmente con stratagemma non diverso dalla Dissertazione sui Sepolcri, Foscolo voleva pubblicare col nome dell'amico Girolamo Federigo Borgno ${ }^{16}$.

I debiti contratti da Foscolo con Virgilio, benché fosse il rappresentante di una tradizione da cui lo scrittore prende progressivamente le distanze, sono importanti ${ }^{17}$. Prova ne sia la presenza tra i suoi libri, per più di un decennio, dell'edizione in quattro volumi dello Heyne stampata a Lipsia, «sumptibus Casparis Fritsch», nel $1787-1789^{18}$, che lo scrittore aveva acquistato a Firenze al principio del $\mathrm{I} 8 \mathrm{O} \mathrm{I}^{\mathrm{I}}{ }^{9}$. L'edizione riproponeva ampliandola quella stampata sempre a Lipsia tra il 1767 e il I775. Heyne offriva a Foscolo un potente strumento esegetico per accostarsi alle Georgiche. Lo prova la presenza, tra i libri di Foscolo, di un'altra edizione virgiliana, la replica procurata dal Pasquali nel 1736 di quella Masuiciana stampata a Leuwarden nel I7I7 da Francesco Halma, acquisita da Foscolo a Milano nel $1802^{20}$. Su di essa, infatti, Foscolo appose varie annotazioni che riguardano in gran parte passi lucreziani e qualche riflessione stilistico-lessicale sul testo delle Georgiche tratta dal commento di Heyne ${ }^{21}$. Il libro II delle

I5. Sul Virgilio di Heyne e sulla sua presenza all'altezza della Chioma ha scritto pagine illuminanti F. Longoni (Foscolo e Virgilio. A proposito di due edizioni virgiliane appartenute a Ugo Foscolo, con postille inedite, "Studi di filologia italiana", LV, I997, pp. I4I-I7I), cui si rinvia per una disanima più particolareggiata.

16. Si veda Il sistema degli Inni esposto da G[irolamo] F[ederigo $B[$ orgno], in EN I, pp. 957-96o.

17. F. Longoni, Foscolo e Virgilio, cit., p. I43. La presenza di Virgilio in Foscolo resta meno studiata rispetto a quella di altri autori classici come Lucrezio, Properzio, Lucano o Giovenale. Si tratta di una lacuna ora in parte colmata, per i Sepolcri, da S. Italia, Virgilio nei «Sepolcri» di Ugo Foscolo: prove di intertestualità, «I fatti e la storia», n.s., V, 20I2, $\mathrm{n}^{\circ} \mathrm{I}$, pp. 9I-II2.

18. P. Virgilii Maronis Opera varietate lectionis et perpetua adnotatione illustrata a Chr. Gottl. Heyne... accedit Index vberrimus. Editio altera emendatior et auctior, Lipsiae, sumtibus Caspari Fritsch, 1787-1789, 4 voll. L'edizione riproponeva ampliandola quella stampata sempre a Lipsia tra il 1767 e il 1775 (su cui si veda ancora F. Longoni, Foscolo e Virgilio, cit., pp. I43-I44) che Foscolo ricorda nel Discorso primo del commento alla Chioma di Berenice.

19. F. Longoni, Foscolo e Virgilio, cit., p. I43. Essa figura ancora tra i libri di Foscolo al momento della partenza per l'esilio, nel marzo del I8Is (cfr. La biblioteca fiorentina del Foscolo nella Biblioteca Marucelliana, premessa di L. Caretti, introduzione, catalogo, appendice di G. Nicoletti, Firenze, Spes, 1978, pp. 92 e 96. Si tratta del $n^{\circ} 9$ della lista $A$, e del $n^{\circ} 29$ della lista B).

20. Ibid., pp. I44-I45. Essa può identificarsi con quella in due volumi restata presso il Pellico nel I8I5 ( $L a$ biblioteca fiorentina, cit., p. I05).

2I. F. Longoni, Foscolo e Virgilio, cit., p. I45. Le annotazioni di Foscolo trovano riscontro nelle tracce di lettura e nelle citazioni lucreziane che lasciò sull'edizione dell'Heyne. Si tratta di Georg. III, 52I, e di De rerum 
Georgiche, quello sulla fertilità delle campagne, che contiene anche la lode all'Italia, è messo in rapporto con il De rerum natura, come nelle Grazie, dove la ricostruzione della nascita e dello sviluppo della civiltà umana rinvia al poema lucreziano. Sulla falsariga di quanto osservava già Franco Longoni per la Chioma, le Georgiche appaiono, in dialogo col poema filosofico lucreziano, il modello di poema didascalico privilegiato da Foscolo per le Grazie. Questo corto circuito non stupisce quando si sa che lo scrittore era convinto che Virgilio avesse tratto linfa vitale per la propria poesia da Lucrezio ${ }^{22}$. Per Foscolo la mediazione formale, stilistica e lessicale del Virgilio lucreziano delle Georgiche, e del suo «ingenium in inveniendis rebus homericum ${ }^{23}$, è essenziale. Benché né l'uno né l'altro potessero rientrare nel rastrematissimo canone dei poeti "primitivi», Foscolo sceglie Virgilio come modello poetico per le Grazie:

Però il poeta rappresenta immagini nuove, per destare affetti lieti alla sua patria contristata dalle vicende politiche: tale dev'essere l'unico scopo della Poesia; e Virgilio adornò nelle Georgiche le arti dell'agricoltura per distorre le menti de' romani dal furore delle guerre civili.

Il miglioramento de' costumi che appare pur sempre generato da le fantasie de' mortali dev'essere l'unico scopo della poesia ${ }^{24}$.

E nel cosiddetto Sommario del Quadernone, alla parte III dell'Inno Secondo, il passaggio di testimone tra Orfeo e Virgilio, raffigurato nel dono della lira, è definito da Foscolo «il più bel dono che le Grazie videro dare all'Italia» ${ }^{25}$.

Il Virgilio delle Georgiche interferisce qui con quello della VI Ecloga, in cui il poeta latino aveva adombrato il sistema epicureo. Rispetto all'esposizione epicurea, Virgilio aveva trattato l'origine degli uomini e della prima età nella quale erano vissuti. Non a caso illustrando le ragioni della scelta, per le Grazie, del modello di una poesia che sia al tempo stesso lirica e didattica Foscolo fa riferimento proprio alla VI Egloga virgiliana:

[...] il fondo del Carme delle Grazie è didattico, e lo stile è tra l'epico e il lirico; per ciò che nel raccontare e questo è l'ufficio principale del puro epico una serie d'avvenimenti l'entusiasmo del poeta li trasforma in altrettante pitture l'una dipendente dall'altra, e formanti un tutto che come nella poesia lirica il lettore può comprendere non tanto nel ricordarsi i fatti narrati, quanto nel rappresentarsi vivamente le immagini,

natura, II, vv. 352-366, brani posti in relazione fra loro nel commento alla Chioma (EN VI, p. 343). Cfr. F. Longoni, Foscolo e Virgilio, cit., p. I45-I46.

22. Ivi, p. I70.

23. P. Virgilii Maronis Opera, cit., vol. I, p. CLXXXI.

24. Si tratta delle note all'Inno primo (EN I, p. IOO3).

25. EN I, p. 994, ma si veda anche p. 997. 
e, gli affetti che ne risultano. A taluni dispiacerà forse questa novità di mescolare il didattico, l'epico, e il lirico, in un solo genere [...]; ma dirò solo che la non è novità $[\ldots]$; e tale fu forse la prima poesia; e per citare un maestro più autorevole a' critici tale è il carme da Virgilio intitolato Sileno, dove con nuove vivissime immagini espose il sistema epicureo nel acanto del vecchio dio, e nelle favole di Pasifae, e di Tereo le passioni sfrenate che turbano la tranquillità dell'animo, unico scopo della filosofia d'Epicuro ${ }^{26}$.

Nelle annotazioni destinate alla prima nota dell'Inno terzo, l'egloga del Sileno è evocata come «divino esempio» di una poesia «lirica» ispirata ai "primitivi principj», in cui l'autore espone il sistema epicureo e, sulla falsariga del commento dello Heyne, Foscolo può affermare che, "oltre a' principi su la formazione del mondo, le favole di Pasifae, e d'altre vittime sacrificatesi alle loro sciagurate passioni, alludono all'opinione d'Epicuro il quale riponeva la beatitudine nella tranquillità dell'anima» ${ }^{27}$.

Quando Foscolo scriveva, da quasi mezzo secolo Heyne era il più influente insegnante, erudito e scrittore classico tedesco ed europeo ${ }^{28}$. Egli si era accostato al mito greco in modo nuovo e originale, individuandovi la più antica storia e la più antica filosofia di un popolo. Sulle orme di Heyne, per Foscolo la poesia era lo strumento per accedere ai miti primordiali, testimoni e custodi del linguaggio simbolico ${ }^{29}$. Non stupisce, allora, che dovendo rivestire del velo candidissimo delle Grazie la trattazione del processo secolare che aveva portato l'uomo «dalla fierezza della barbarie alla raffinatissima civiltà» ${ }^{30}$, e quella del ruolo che costruendo e promuovendo il legame sociale vi avevano svolto le arti letterarie, Foscolo facesse ancora una volta appello al grande filologo tedesco e al suo autore prediletto, Virgilio.

26. EN I, pp. 958-959.

27. EN I, p. 972.

28. Nel Commento alla "Chioma di Berenice», Foscolo definisce Heyne un commentatore «chiaro e fortunato" (EN VI, p. 278).

29. EN VI, p. 392. Su Foscolo e il mito si segnalano i due saggi di M. Salvini, Le riflessioni foscoliane sul mito, "Otto-Novecento", XIX, I995, n 6, pp. 3I-5I, e di E. Selmi, Mito e allegoria nella poetica del Foscolo, «La Rassegna della Letteratura italiana», XCVIII, I994, n I, pp. 76-95. Questi due brevi saggi si accostano al volume di L. Derla, L'isola, il velo e l'ara. Allegoria e mito nella poesia di Ugo Foscolo, Genova, Edizioni Culturali Internazionali, 1984, cui va riconosciuto il merito di aver richiamato l'attenzione su un aspetto della riflessione foscoliana che era restato fino ad allora largamente in ombra.

30. Come afferma nell'orazione Dell'origine e dell'ufficio della letteratura. Cfr. EN VII, p. 22. 